\title{
Celiac disease is overrepresented in patients with constipation
}

\author{
Doença celíaca é super-representada em pacientes com constipação \\ Rolf A. A. Pelleboer ${ }^{1}$, Rob L. H. Janssen2, Judith M. Deckers-Kocken ${ }^{3}$, \\ Edward Wouters ${ }^{4}$, Annemieke C. Nissen ${ }^{5}$, Werner E. A. Bolz ${ }^{6}$, Walther E. Tjon A. Ten ${ }^{7}$, \\ Cathelijne van der Feen ${ }^{8}$, Koen J. Oosterhuis ${ }^{9}$, Mechelien H. Rövekamp ${ }^{10}$, \\ Peter G. J. Nikkels ${ }^{11}$, Roderick H. J. Houwen ${ }^{12}$
}

\section{Resumo}

Objetivo: Tem sido sugerido que pacientes com constipação sejam triados para doença celíaca. Da mesma forma, recomenda-se a investigação desses pacientes para hipotiroidismo e hipercalcemia. Contudo, nenhuma evidência para essas recomendações está disponível até o momento. Assim, propusemos-nos determinar a prevalência de doença celíaca, hipotiroidismo e hipercalcemia em crianças com constipação.

Métodos: Estudo de coorte prospectivo com 370 pacientes consecutivos que preencheram os critérios de Roma III para constipação. Esses pacientes foram encaminhados por um clínico geral a um pediatra devido ao fracasso no tratamento com laxantes.

Resultados: A biópsia comprovou doença celíaca em sete desses pacientes. Isso é significativamente mais alto $(p<0,001)$ do que a prevalência de 1:198 de doença celíaca nos Países Baixos. Dois pacientes tinham tiroidite autoimune. Nenhum paciente tinha hipercalcemia.

Conclusões: Conclui-se que a doença celíaca é significativamente super-representada em pacientes com constipação encaminhados por um clínico geral a um pediatra devido ao fracasso no tratamento com laxantes. Todos esses pacientes devem, portanto, ser triados para doença celíaca.

J Pediatr (Rio J). 2012;88(2):173-6: Doença celíaca, constipação, hipotiroidismo, hipercalcemia.

\section{Abstract}

Objective: It is suggested that patients with constipation should be screened for celiac disease. Similarly, it is recommended to investigate these patients for hypothyroidism and hypercalcemia. However, no evidence for these recommendations is available so far. We therefore set out to determine the prevalence of celiac disease, hypothyroidism, and hypercalcemia in children with constipation.

Methods: Prospective cohort study of 370 consecutive patients who met the Rome III criteria for constipation. These patients were referred by a general practitioner to a pediatrician because of failure of laxative treatment.

Results: Seven of these patients had biopsy-proven celiac disease. This is significantly higher $(p<0.001)$ than the $1: 198$ prevalence of celiac disease in the Netherlands. Two patients had auto-immune thyroiditis. No patient had hypercalcemia.

Conclusions: We conclude that celiac disease is significantly overrepresented in patients with constipation who are referred by a general practitioner to a pediatrician because of failure of laxative treatment. All such patients should, therefore, be screened for celiac disease.

J Pediatr (Rio J). 2012;88(2):173-6: Celiac disease, constipation, hypothyroidism, hypercalcemia.

1. MD. Pediatric gastroenterologist, Department of Pediatrics, Catharina Hospital, Eindhoven, Holanda.

2. MD. General physician, Department of Pediatrics, Catharina Hospital, Eindhoven, Holanda.

3. MD. PhD. Pediatric gastroenterologist, Flevo Hospital Almere, at present 's-Hertogenbosch, Holanda.

4. MD. General physician, Flevo Hospital Almere, Holanda.

5. MD. Pediatrician, St. Elisabeth Hospital, Tilburg, Holanda.

6. MD. PhD. Pediatrician, Elkerliek Hospital, Helmond, Holanda.

7. MD. PhD. Pediatric gastroenterologist, Máxima Medical Center, Veldhoven, Holanda.

8. MD. Pediatrician. Fellow pediatric gastroenterology, Wilhelmina Children's Hospital, University Medical Center, Utrecht, Holanda.

9. MD. Pediatrician, St. Jansdal Hospital, Harderwijk, Holanda.

10. MD. PhD. Pediatrician, Gelre Hospital, Apeldoorn, Holanda.

11. MD. PhD. Pediatric pathologist, Department of Pathology, Wilhelmina Children's Hospital, University Medical Center, Utrecht, Holanda.

12. MD. PhD. Pediatric gastroenterologist, Wilhelmina Children's Hospital, University Medical Center, Utrecht, Holanda.

Não foram declarados conflitos de interesse associados à publicação deste artigo.

Apoio financeiro: este trabalho foi financiado pela Fundação Científica do Catharina Hospital, Eindhoven, Países Baixos (nr. WF 05-08).

Como citar este artigo: Pelleboer RA, Janssen RL, Deckers-Kocken JM, Wouters E, Nissen AC, Bolz WE, et al. Celiac disease is overrepresented in patients with constipation. J Pediatr (Rio J). 2012;88(2):173-6.

Artigo submetido em 22.07.11, aceito em 06.10.11.

http://dx.doi.org/10.2223/JPED.2155 


\section{Introdução}

A doença celíaca (DC), uma doença inflamatória crônica caracterizada por vilosidades achatadas na mucosa do intestino delgado, é induzida em pessoas geneticamente susceptíveis pela ingestão de proteínas do trigo, centeio e cevada que contêm glúten ${ }^{1,2}$. A apresentação clássica se dá com diarreia crônica, distensão abdominal e déficit de crescimento. Contudo, outros sintomas, como dor abdominal, peso para altura inferior ao percentil 10 e lassidão ou anemia são encontrados com frequência cada vez maior ${ }^{3}$. Constipação também pode ser o sintoma de apresentação de DC, embora a frequência desse fenômeno seja desconhecida ${ }^{4}$. Todavia, a Sociedade Norte-Americana de Gastroenterologia Pediátrica, Hepatologia e Nutrição (NASPGHAN) sugere o teste para DC em crianças constipadas quando o tratamento oral com laxantes não obtiver sucesso ${ }^{5}$. Além disso, recomenda-se o teste para hipotiroidismo e hipercalcemia para esse grupo ${ }^{5}$. Contudo, é necessária evidência formal de que a DC é superrepresentada em pacientes com constipação. Da mesma forma, é desconhecida a frequência de hipotiroidismo ou hipercalcemia em pacientes pediátricos com constipação.

O objetivo do presente estudo foi, portanto, determinar a prevalência de DC em crianças com constipação que foram encaminhadas por um médico de atenção primária a um pediatra. Ao mesmo tempo, esses pacientes foram triados para hipotiroidismo e hipercalcemia.

\section{Pacientes e métodos}

Entre outubro de 2006 e outubro de 2008, foram incluídos 370 pacientes consecutivos prospectivamente com constipação. Oito centros pediátricos nos Países Baixos participaram: Catharina Hospital Eindhoven, Flevo Hospital Almere, St. Elisabeth Hospital Tilburg, Elkerliek Hospital Helmond, Máxima Medical Center Veldhoven, St. Jansdal Hospital Harderwijk, Gelre Hospital Apeldoorn e Wilhelmina Children's Hospital Utrecht. O estudo foi feito de acordo com as normas dos comitês de ética institucionais. O Comitê de Ética e Revisão Médica (Medical Ethics Review Committee, METC) do Catharina Hospital aprovou o estudo acima mencionado. O METC revisou os protocolos de pesquisa de acordo com as regras estabelecidas no Estatuto para Pesquisas Médicas Envolvendo Seres Humanos (Medical Research Involving Human Subjects Act,WMO).

Os pacientes incluídos foram encaminhados por um clínico geral a um pediatra devido ao fracasso inicial do tratamento com laxantes para constipação. Todos os 370 pacientes incluídos preencheram os critérios de Roma III 6,7 para constipação: pacientes entre 1 e 4 anos tinham que ter pelo menos dois dos seguintes sintomas por 1 mês: duas ou menos evacuações por semana; pelo menos um episódio por semana de incontinência depois de aprender a usar o banheiro; histórico de retenção excessiva de fezes; histórico de evacuação dolorosa ou difícil; presença de uma grande massa fecal no reto e histórico de fezes de grande diâmetro que podem obstruir o vaso sanitário; pacientes de 4 a 18 anos tinham que preencher esses critérios por pelo menos 2 meses ao invés de 1 mês e, também, tinham que ter critérios insuficientes para o diagnóstico de Síndrome do Intestino Irritável (SII). Todos os pacientes tinham entre 1 e 18 anos e tiveram pelo menos 3 meses de ingestão de glúten. Uma triagem primária para DC for feita através da determinação da IgA sérica total e da transglutaminase tecidual humana IgA (Kit Celikey tTG Elisa, Pharmacia and Upjohn Diagnostics, Friburgo, Alemanha). Em todos os pacientes com uma IgA-tTG anormal, ou uma IgA sérica baixa, foi realizada uma biópsia do intestino delgado. A avaliação final foi feita por um único e experiente patologista pediátrico (PN) e as biópsias foram avaliadas de acordo com os estágios de Marsh ${ }^{8}$. Nessa classificação, um número maior de linfócitos intraepiteliais é visto no estágio 1 ; no estágio 2 , além disso, percebe-se um aumento na profundidade cripta, mas sem vilosidades achatadas. No estágio 3, percebe-se um número maior de linfócitos intraepiteliais e um aumento da profundidade cripta, assim como uma redução da altura das vilosidades, que é graduada em a, b e c, com c significando atrofia vilositária total. Somente pacientes com Marsh 3, tanto com a, b ou c, foram julgados como tendo DC. Além de avaliar para DC, também foram determinados o cálcio sérico, o nível sérico de T4 livre e o nível sérico de TSH.

Para determinar se a frequência de DC é de fato maior entre pacientes com constipação, foi utilizado o teste exato de Fisher bicaudal.

\section{Resultados}

No intervalo de 2 anos entre outubro de 2006 e outubro de 2008, foram incluídos um total de 370 pacientes com constipação. A biópsia comprovou DC em sete desses pacientes (Marsh 3, Tabela 1). Isso é significativamente maior ( $p<0,001$ ) do que a prevalência de 1:198 de DC nos Países Baixos, como determinado em uma pesquisa com 6.127 crianças holandesas ${ }^{9}$. Tanto o crescimento estatural como ponderal dessas sete crianças eram normais antes da biópsia. Laxantes não se fizeram mais necessários depois do início de uma dieta livre de glúten em duas das sete crianças; em três, os laxantes puderam ser reduzidos; e duas precisaram da mesma quantidade de laxantes.

Em duas pacientes (ambas meninas, 8 e 9 anos de idade) foi encontrado um baixo nível sérico de T4 livre e um maior nível sérico de TSH. Testes subsequentes demonstraram em ambas títulos aumentados de anticorpos antiperoxidase (TPO), de modo que foi feito o diagnóstico de tireoidite autoimune. Constipação por três meses antes do encaminhamento foi a única queixa. Não houve outros sinais clínicos de hipotireoidismo. Foi iniciado tratamento com tiroxina e um laxante. Os laxantes puderam ser suspensos depois de algum tempo em ambas as pacientes. Diagnóstico de hipercalcemia não foi feito em nenhum paciente.

\section{Discussão}

Nosso estudo realmente mostrou uma prevalência maior de DC em pacientes pediátricos com constipação encaminha- 
Tabela 1 - Pacientes com doença celíaca que apresentaram constipação

\begin{tabular}{|c|c|c|c|}
\hline Sexo & $\begin{array}{c}\text { Idade ao ser } \\
\text { diagnosticado } \\
\text { (anos) }\end{array}$ & Biópsia & $\begin{array}{l}\text { Sintomas e tratamento de constipação } \\
\text { depois de iniciar dieta livre de glúten }\end{array}$ \\
\hline Masculino & $11 / 4$ & Marsh 3b & Sem mais constipação; sem uso de laxante \\
\hline Feminino & 12 & Marsh 3b & Sem mais constipação; sem uso de laxante \\
\hline Masculino & 3 & Marsh 3b & Constipação melhor; precisa de menos laxante \\
\hline Feminino & $31 / 2$ & Marsh $3 a$ & Constipação melhor; precisa de menos laxante \\
\hline Feminino & 8 & Marsh $3 a$ & Constipação melhor; precisa de menos laxante \\
\hline Masculino & $11 / 2$ & Marsh 3c & Ainda constipado; precisa da mesma quantidade de laxante \\
\hline Masculino & $21 / 4$ & Marsh 3b & Ainda constipado; precisa da mesma quantidade de laxante \\
\hline
\end{tabular}

dos por um clínico geral a um pediatra devido ao fracasso inicial de tratamento com laxantes. Isso significa que em cada criança com constipação e sem resposta ao tratamento, a DC deve ser excluída, assim como outras causas mais conhecidas de constipação (ex.: doença de Hirschsprung). A causa dessa relação entre DC e constipação não é clara, mas sugerimos que a inflamação da mucosa na DC pode afetar a motilidade intestinal normal, como é visto em uma forma extrema em crise celíaca ${ }^{10}$.

Duas de nossas pacientes com constipação infantil tinham tireoidite autoimune. Embora DC e tireoidite autoimune estejam relacionadas, nenhuma dessas duas crianças tinha DC. Além disso, tireoidite autoimune em crianças é bastante rara, com uma prevalência de $0,3 \%{ }^{11}$. Então, a frequência que foi encontrada (2/370) parece ser maior, ainda que não significativamente. Embora o número de pacientes incluídos em nosso estudo tenha sido feito para encontrar uma resposta à pergunta se a DC é ou não super-representada em pacientes pediátricos com constipação, o poder foi insuficiente para detectar uma super-representação de problemas de tiróide. Como ambos os nossos pacientes estavam livres de sintoma, além de constipação, a triagem para problemas de tireóide pode ser considerada para qualquer criança com constipação atendida por um pediatra.

Nenhuma criança em nosso estudo tinha hipercalcemia, que poderia induzir constipação por uma excitabilidade neuromuscular reduzida devida a níveis altos de cálcio ${ }^{12}$. Contudo, apenas uma criança com hipercalcemia relacionada à constipação foi descrita até o momento ${ }^{13}$. Portanto, a baixa frequência encontrada (0/370) não é uma surpresa.

Em conclusão, DC é significativamente super-representada em pacientes com constipação encaminhados por um clíni- co geral a um pediatra devido ao fracasso do tratamento com laxantes. Todos os pacientes deveriam, portanto, ser triados para DC.

\section{Referências}

1. Di Sabatino A, Corazza GR. Coeliac disease. Lancet. 2009;373:1480-93.

2. Castro-Antunes MM, Magalhães R, Nobre JM, Duarte BP, Silva GA. Celiac disease in first-degree relatives of patients. J Pediatr (Rio J). 2010;86:331-6.

3. Steens RF, Csizmadia CG, George EK, Ninaber MK, Hira Sing RA, Mearin ML. A national prospective study on childhood celiac disease in the Netherlands 1993-2000: an increasing recognition and a changing clinical picture. J Pediatr. 2005;147:239-43.

4. Egan-Mitchell B, McNicholl B. Constipation in childhood coeliac disease. Arch Dis Child. 1972;47:238-40.

5. Baker SS, Liptak GS, Colletti RB, Croffie JM, Di Lorenzo C, Ector $\mathrm{W}$, et al. Constipation in infants and children: evaluation and treatment. A medical position statement of the North American Society for Pediatric Gastroenterology and Nutrition. J Pediatr Gastroenterol Nutr. 1999;29:612-26.

6. Hyman PE, Milla PJ, Benninga MA, Davidson GP, Fleisher DF, Taminiau J. Childhood functional gastrointestinal disorders: neonate/toddler. Gastroenterology. 2006;130:1519-26.

7. Rasquin A, Di Lorenzo C, Forbes D, Guiraldes E, Hyams JS, Staiano A, et al. Childhood functional gastrointestinal disorders: child/ adolescent. Gastroenterology. 2006;130:1527-37.

8. Marsh MN. Gluten, major histocompatibility complex, and the small intestine. A molecular and immunobiologic approach to the spectrum of gluten sensitivity ('celiac sprue'). Gastroenterology. 1992;102:330-54.

9. Csizmadia CG, Mearin ML, von Blomberg BM, Brand R, VerlooveVanhorick SP. An iceberg of childhood coeliac disease in the Netherlands. Lancet. 1999;353:813-4. 
10. Mones RL, Atienza KV, Youssef NN, Verga B, Mercer GO, Rosh JR. Celiac crisis in the modern era. J Pediatr Gastroenterol Nutr. 2007;45:480-83.

11. LaFranchi S. Hypothyroidism. In: Kliegman RM, Stanton BF, St Geme III JW, Schor NF, Behrman RE, eds. Nelson textbook of pediatrics. 19th ed. Philadelphia, PA: Saunders Elsevier; 2011. p. 1903-4.

12. Ebert EC. The parathyroids and the gut. J Clin Gastroenterol. 2010;44:479-82.
13. Lillquist K, Illum N, Jacobsen BB, Lockwood K. Primary hyperparathyroidism in infancy associated with familiar hypocalciuric hypercalcemia. Acta Pediatr Scand. 1983;72:625-9.

Correspondência:

Rolf Pelleboer

Department of Paediatrics, Catharina Hospital

PO Box 1350,5602 ZA - Eindhoven, Holanda

E-mail: rolf.pelleboer@cze.nl 